Relations industrielles

Industrial Relations

\title{
Tapscott, Don, and Art Caston. Paradigm Shift. The New Promise of Information Technology
}

\section{Rolland Hurtubise}

Volume 48, numéro 3, 1993

URI : https://id.erudit.org/iderudit/050881ar

DOI : https://doi.org/10.7202/050881ar

Aller au sommaire du numéro

Éditeur(s)

Département des relations industrielles de l'Université Laval

ISSN

0034-379X (imprimé)

1703-8138 (numérique)

Découvrir la revue

Citer ce compte rendu

Hurtubise, R. (1993). Compte rendu de [Tapscott, Don, and Art Caston. Paradigm Shift. The New Promise of Information Technology]. Relations industrielles / Industrial Relations, 48(3), 570-577.

https://doi.org/10.7202/050881ar

Tous droits réservés (C) Département des relations industrielles de l'Université Laval, 1993
Ce document est protégé par la loi sur le droit d'auteur. L'utilisation des services d'Érudit (y compris la reproduction) est assujettie à sa politique d'utilisation que vous pouvez consulter en ligne.

https://apropos.erudit.org/fr/usagers/politique-dutilisation/ 
The volume as a whole, and the editor in particular, have succeeded in making a valuable contribution to the industrial relations literature. Contemporary employers in both the union and non-union sector are facing and will continue to face difficult labour issues because of structural change. This book is tremendously useful because of the historical perspective it provides. Structural change is not new, and the volume reveals the wide variety of ways of adapting to change that have been used in the past.

Overall, my enthusiasm for the book is high. The editor has done a masterful job of making a coherent whole out of eight very different studies. Each contributor, in turn, has produced a well researched and well written chapter.

The only major criticism I have is that I believe that the book does not adequately address issues surrounding how employers have dealt with women and with people of colour. Labour history teaches us that union policies toward these two groups were not always the same as policies directed towards white males; is it not reasonable to assume similar differentiation by employers? If so, it would be instructive to learn more about how and why employer policies differentiated these groups. Perhaps this issue could be the subject of as fine a volume as that reviewed here!

Norman A. Solomon

University of Windsor

Paradigm Shift. The New Promise of Information Technology, by Don Tapscott and Art Caston, New York, McGraw-Hill, 1993, 337 p., ISBN : 0-07-062857-2

Un vade-mecum?

Tout est paradigmatique dans ce livre. Tout est transfert (shift).

$\mathrm{Si}$, au moment de lire ces lignes, cet ouvrage n'est pas encore devenu un bestseller, c'est que cela ne tardera pas! Il est étonnant de voir le nombre de personnes qui "voyagent " avec ce livre et qui profite d'occasions pour le montrer à un collègue ( Vous avez vu ce livre? ») ou, encore, pour en lire quelques pages. On a l'impression que plusieurs managers en ont fait leur livre de chevet.

Il faut admettre que Paradigm Shift, dont le sous-titre se traduit par : La nouvelle promesse des technologies de l'information vise le manager. Écrit par deux viceprésidents de la célèbre firme canadienne de consultants de management et de technologie de l'information : Ducros, Meilleurs et Roy (DMR), il se veut le fruit de recherches réalisées auprès de milliers d'organisations américaines, canadiennes et étrangères. S'agit-il du vade-mecum des managers en la matière de technologie de l'information (TI)?

\section{Un livre condensé qui se veut englobant}

Les premières lignes du livre risquent d'apeurer le lecteur. Dès sa préface on peut lire qu'une mutation fondamentale est en cours dans le monde des affaires quant à la nature et à l'instauration de la technologie et que cette évolution risque d'avoir des 
incidences sur l'organisation du lecteur. Qui plus est, celui-ci apprend que personne à ce jour n'a su complètement articuler ce changement! Dès le départ, le lecteur-manager a donc droit de s'interroger s'il s'agit d'un autre texte de révélations... selon un autre saint du management.

Dès le début, il est question de quatre transferts (shifts) de paradigme reliès à la nouvelle technologie, au nouvel environnement des affaires (business), au nouvel ordre géopolitique et, il ne faudra pas l'oublier, à la nouvelle entreprise. Dans ce contexte, les auteurs ont signalé (rapidement) que la notion de paradigme n'est plus celle définie par les dictionnaires, mais qu'elle communique désormais des concepts de modèle, de cadre, de manière de penser, de schème pour comprendre la réalité.

Le chapitre introductif décrit le nouvel environnement des affaires (business toujours!) qui, caractérisé par sept « poussées » (thrusts), comprend la productivité des travailleurs du savoir et des services, la qualité, la rapidité de réaction, la mondialisation, la contractualisation (outsourcing), le partenariat et la responsabilité sociale et environnementale. Afin de bien situer le tout, les auteurs identifient deux types d'organisations : l'« hiérarchie fermée » et statique, celle du management ferme, organisation pliée sur elle-même, axée sur l'investissement en capital, le contrôle managériel et l'importance des positions hiérarchiques; et le « réseau » ouvert, dynamique et changeante, celle du leadership, qui est plus humaine et axée sur l'information et les équipes de professionnels engagés. Rapidement - mais très rapidement ! - cette même introduction transporte le lecteur vers trois transferts critiques de l'instauration de la TI : de l'informatique personnelle à l'informatique du groupe de travail ; des systèmes insulaires aux systèmes intégrés; de l'informatique interne à l'informatique interentreprise. Il s'agit d'une trop brève tentative de relier ces transferts au double plan organisationnel et managériel. D'ailleurs, il faut s'interroger à savoir s'il y a adéquation entre ces trois transferts et les huit transferts technologiques critiques suivants, eux-mêmes subdivisés en trois volets, qui distinguent les systèmes (organisations ?) de «l'ère I » de ceux de «l'ère II ». Sous le volet de l'informatique réseau, deux transferts sont exposés : un premier relié au traitement (la transition des semi-conducteurs traditionnels vers les microprocesseurs) et un second touchant les systèmes proprement dits (la transition des systèmes « hôtes » ou centralisés vers les réseaux). Sous le volet des systèmes ouverts, trois transferts sont énumérés : la transition des logiciels des fournisseurs vers les normes qui rendent les logiciels indépendants (des fournisseurs) ; la transition des représentations informationnelles séparées (donnée, texte, voix, image) vers le multimédia ; la transition du contrôle de comptes par un fournisseur vers un partenariat multifournisseur. Finalement, sous le volet de la révolution industrielle dans le domaine des logiciels, trois derniers transferts sont expliqués : la transition des logiciels faits sur mesure vers l'usinage ou l'ingénierie des logiciels; la transition de l'interface utilisateur alphanumérique vers le graphique; la transition des applications indépendantes vers les applications intégrées.

Le reste du livre est divisé en trois parties intitulées, respectivement : " La promesse ", «Le paradigme de la nouvelle technologie » et «La Transition ».

C'est à la partie « La promesse » que les auteurs explicitent, dans autant de chapitres, les trois promesses liées aux trois transferts critiques préalablement signalés pour l'instauration de la TI. La première promesse implique la reconception du processus des affaires (business toujours!) et vise l'équipe à haute performance qui se sert de 
l'informatique de groupe. La seconde suggère la transformation de l'organisation et touche l'organisation intégrée qui s'appuie sur les systèmes intégrés. Finalement, survient la promesse de l'entreprise élargie qui utilise l'informatique interentreprise à la suite de la refonte de ses relations extérieures.

Le chapitre sur l'informatique du groupe de travail, l'équipe à haute performance, ne manque pas de convaincre. Les auteurs y décrivent les postes de travail multifonctionnels reliés en réseau et ils évoquent la nécessité de reconcevoir les tâches, les processus et les structures de travail. $\dot{A}$ cette rubrique, outre les objectifs d'optimiser les communications et d'éliminer les activités non productives, il est question d'améliorer la création participative, de renforcer le remue-méninges et de raffermir la prise de décisions (Hélas! Le sujet de la prise de décisions est trop peu apostrophé dans ce livre.). Les bénéfices qui découlent de l'instauration de tels groupes de travail sont précisés: les « gains en temps » et l'amélioration de la qualité du travail et des extrants (produits, services). Fait à noter, à la fin de ce chapitre, les auteurs signalent non seulement le transfert menant à la reconception du système de travail au complet, mais ils soulignent l'importance du leadership requis afin de modifier les façons de travailler.

L'organisation intégrée, la seconde promesse, est le thème d'un chapitre plutôt court. On discute des systèmes de l'ère I, composés d'îlots de technologie séparés et isolés qui reflètent le manque d'intégration, ainsi que des systèmes de l'ère II, où il est question des qualités des organisations et des systèmes intégrés. Encore une fois, en discutant de transferts, les auteurs repassent sur l'importance d'une restructuration organisationnelle axée non pas sur la diminution des coûts, mais plutôt sur l'efficacité de l'entreprise afin de pouvoir instaurer l'environnement informatique intégré.

La troisième promesse (un autre court chapitre!) est reliée à l'entreprise élargie. Ici, les transferts retenus méritent une attention toute particulière. Outre la transition des îlots de technologie dans l'entreprise vers l'informatique interorganisationnelle, on discute de valeurs (organisationnelles) et du transit d'une simple chaîne (interne) vers un réseau de valeurs qui implique les fournisseurs, les consommateurs, les groupes « voisins » et même les compétiteurs! Le tout est fondé sur la technologie et sur les communications électroniques au travers le réseau de valeurs. Qui plus est, les auteurs signalent que l'entreprise risque de devenir non pas uniquement un acheteur, mais aussi un vendeur d'information.

La deuxième partie du livre traite du paradigme de la nouvelle technologie et des trois transferts technologiques clés qui permettent d'accéder à l'équipe haute performance, à l'organisation intégrée et à l'entreprise élargie. Un premier chapitre détaille le premier transfert de l'informatique hôte de l'ère I vers l'informatique réseau, de type client/serveur, de l'ère II. Il fait aussi état des avantages de cette dernière en termes d'adéquation opérationnelle, de spécialisation des sous-systèmes, de diminution des coûts et, bien sûr, d'intégration, de flexibilité et d'innovation. Un deuxième chapitre parle du transfert des systèmes à propriétaire unique vers les systèmes ouverts. Ici, il est question de l'importance de la normalisation et du fait que les systèmes ouverts réduisent les coûts, offrent davantage d'indépendance envers les fournisseurs et, somme toute, accordent un déplacement plus accessible vers la nouvelle technologie. Le troisième chapitre évoque le transfert relié à la révolution industrielle des logiciels et le fait que la conception de ceux-ci est passée d'un art (métier) à une ingénierie (usine). Cette 
transition est caractérisée, notamment, par l'apparition de logiciels de type « Lego » et de l'industrie des modules de logiciel, de l'interface graphique pour utilisateur, de la conception de logiciels assistée par ordinateur et du phénomène qui permet à l'utilisateur de devenir lui-même programmeur.

En exposant les principaux transferts reliés à l'instauration de la TI et les transferts technologiques proprement dits, il est possible que les deux premières parties de Paradigm Shift aient surtout intéressé le lecteur fasciné par les systèmes. Voilà que la troisième partie cherche à capter l'attention du lecteur plus préoccupé par l'organisation et les considérations managérielles. Intitulé " La transition ", elle expose les transferts correspondants particuliers à la manière d'atteindre l'ère II, qualifiée de nouvelle. De fait, les derniers chapitres du livre exposent un cadre de transition vers l'ère II. Ce cadre comprend deux axes. Le premier repose sur quatre plateaux (sic) : « réimager » ou atteindre une vision, réorganiser ou structurer la solution, réaliser ou développer et déployer, et renouveler ou améliorer constamment. En annonçant la fin du plan stratégique organisationnel de la TI (traditionnel - ère I), les auteurs offrent des lignes directrices reliées à autant de transferts en matière de planification qui conduisent vers l'ère Il, celle de «l'action d'apprentissage continue ». En découle l'organisation qui verra sa planification de la TI intimement liée et intégrée, autant à l'interne qu'à l'externe, à la stratégie business (toujours!). Fondée sur le modèle client/serveur, plutôt que sur l'organigramme, l'entreprise de l'ère II s'adonne au traitement de l'information multimédia et à l'atteinte de résultats à très court terme. D'ailleurs, les auteurs recommandent de ne plus entrevoir l'organisation par l'entremise de son organigramme, mais plutôt en terme de logical service units (LSU), ces composantes fonctionnelles fondamentales, indépendantes de la structure organisationnelle, des ressources humaines et technologiques employées, qui décrivent les activités organisationnelles : planification, production, support, ventes, etc. Ce sont les LSU qui servent à réaliser des modèles organisationnels, la réingénierie du travail et des architectures de TI.

Toujours selon les auteurs, ce qui importe dans un premier temps, c'est l'atteinte d'une vision. Ils suggèrent au manager d'initier un processus d'apprentissage, de « réimager » son monde, d'identifier des poussées (thrusts) stratégiques, de "réimager » son organisation selon un modèle business et, finalement, d'établir des principes business pour la réingénierie du travail, la TI et la fonction des systèmes d'information. C'est ainsi que se précise le second axe du cadre de transition qui comporte trois volets : la réingénierie des affaires (business toujours!), le réoutillage de la TI et le réalignement de la fonction des systèmes d'informations (SI).

Le premier volet vise à établir un pont entre la "nouvelle technologie » et la " nouvelle entreprise ». Une entrée en matière persuasive met en relief dix thèmes technologiques (systèmes ouverts, interconnexion, informatique distribuée, temps réel, traitement coopératif, protocoles réseau de type égal-à-égal, " modularité " architecturale, spécialisation des plates-formes, convivialité, réseau global) qui sont appariés à dix thèmes organisationnels (ouverture, intégration, responsabilisation, urgence, coopération, engagement, indépendance organisationnelle, spécialisation des habiletés/création des compétences, accessibilité, indépendance de temps et d'espace). Qui plus est, trente principes de la TI sont énumérés. Ceux-ci, susceptibles de faciliter la réingénierie du travail, sont pour la plupart reliés aux tâches, à la clientèle, au management et à 
l'information. Au sujet de la réingénierie du travail, des conseils pratiques, quasi personnels, sont offerts au lecteur : "Rappelez-vous que le programme de reconception des systèmes de travail doit appartenir et être contrôlé par le personnel des affaires et non pas par les spécialistes des SI. Visez les solutions rapides. Rappelez-vous que les communications avec les personnes impliquées constituent le gage de réussite. Obtenez une méthode et une expertise externe. Impliquez le personnel des SI. Concentrez-vous sur les bénéfices. Mesurez! Pensez grand, commencez petit! ". Le lien entre le premier axe du cadre de transition et les plateaux rappelle l'importance, au niveau de la réingénierie du travail, d'atteindre une nouvelle vision, de structurer un nouveau système de travail, d'implanter les programmes et de prolonger l'implantation (l'amélioration constante). Une importance est accordée aux types d'activités qui peuvent être combinés afin de modeler ce qui se passe dans une unité de travail composée de travailleurs des connaissances ou des services, ainsi qu'aux implications de la reconception d'un nouveau système de travail.

Le deuxième volet du deuxième axe du cadre de transition est celui du réoutillage de la TI qui implique cinq perspectives de la nouvelle architecture de la TI (celles des affaires, du travail, de l'information, des applications et de la technologie), ainsi que des catégories de principes architecturaux reliés à l'organisation du travail et aux architectures des applications, de l'information et de la technologie. Outre la présentation d'une vingtaine d'environnements d'applications, allant de la saisie des données, en passant par l'appui à la décision pour se terminer à la télédiffusion, les auteurs énumèrent les services pour appuyer l'environnement des applications : le management de l'interface utilisateur, le management de l'information, le management des transactions, les systèmes d'exploitation (pas de management ici ?) et le management des communications. Ils décrivent ensuite les plates-formes technologiques, c'est-à-dire, le matériel client/ serveur requis pour appuyer les applications technologiques, dont les postes de travail intelligents, les serveurs de groupes de travail, de directions d'entreprises et les serveurs externes, tous reliés par réseau local, autocommutateur local et réseau prolongé. Le chapitre en cause se termine par l'exposé de dix lignes directrices pour atteindre l'architecture technologique de l'ère Il. Celles-ci sont présentées selon le format, encore une fois, de conseils pratiques personnels : "Accentuez le processus de planification architecturale et non pas le plan. Assurez-vous que la vision architecturale appartienne conjointement à la fonction des SI et aux unités des affaires. Assurez-vous que la planification architecturale soit commandée par les affaires et non pas par la technologie. Concentrezvous sur la migration et non seulement sur l'architecture ciblée. Concevez en vue du changement continu. Concentrez-vous sur l'action et la livraison de résultats à court terme. Créer un processus de réalisations rapides. Attardez-vous sur la livraison véritable des systèmes. Utilisez les cinq perspectives architecturales comme cadre architectural. Faites-le!"

Le troisième et dernier volet du deuxième axe du cadre de transition est celui du réalignement de la fonction des SI. Les auteurs rappellent les quatre " plateaux » du premier axe et mettent en évidence huit symptômes d'un problème d'alignement organisationnel face aux SI : les conflits internes continus, les plaintes touchant le rendement de la fonction des SI, le manque d'une vision à l'échelle organisationnelle, le déclin de la compétitivité, le manque d'intérêt de la part des unités des affaires ou du management 
supérieur quant à l'emploi efficace de la TI, une base d'habiletés déficiente ou inadaptée, le roulement élevé des professionnels des SI et la duplication quant au développement de systèmes. Le premier plateau du premier axe du cadre de transition est soutenu par douze principes d'alignement directement liés pour la plupart à la structure des SI. Au fait, cinq structures pour aligner les SI avec l'organisation sont proposées : la dispersion des ressources des SI, leur centralisation, leur décentralisation, l'établissement d'un centre de profit ou d'une compagnie séparée et la contractualisation (outsourcing) des SI. Finalement, interprétée en termes de trois poussées (thrusts), une synthèse illustre la transition vers l'organisation des SI de l'ère II en termes, premièrement, de la réingénierie de l'entreprise (de l'hiérarchie fermée vers l'organisation ouverte en réseau), deuxièmement, du réoutillage de la technologie (de l'informatique hôte, de propriétaire vers l'informatique ouverte, réseau) et, troisièmement, du réalignement de la fonction des SI (de la forteresse centralisée vers le facilitateur dispersé).

Le tout demier chapitre du livre philosophe de leadership et du « pont " de transition de l'ère I à l'ère II. Les auteurs répètent les composantes de leur cadre de transition et comment il est primordial de "réimager ", réorganiser, réaliser et renouveler l'organisation des affaires (réingénierie), l'infrastructure de la TI (réoutillage) et l'organisation des SI (réalignement). Encore une fois, des conseils sont offerts (beaucoup d'importance accordée à Citibank et aux manières de mieux apprécier et de s'éduquer face au nouveau paradigme). En guise de conclusion, les auteurs signalent le fait que les ordinateurs deviennent des commodités, que les revenus et les profits se déplacent vers les services et que l'avenir de la technologie devient à caractère coopératif. Ils s'interrogent sur l'avenir de certaines facettes du monde, en situant le tout face à la transformation de l'industrie informatique, au leadership en la matière et aux possibilités de la TI ressenties par les clientèles. Ils raisonnent en termes de compétitivité entre les nations, leurs stratégies et l'éthique (sujets qu'ils admettent appartenir à un autre livre... fort heureusement !). Ils arguent que les nations dont les industries sauront réaliser le transfert paradigmatique deviendront productives et prospères. $\grave{A}$ un niveau organisationnel et managériel, les auteurs citent DMR en exemple (comment faire autrement!) qui, afin de donner l'exemple, a dû se centrer sur ses professionnels - les plus proches des clients! - plutôt que sur ses managers. Ils soulignent également le danger relié à la mauvaise utilisation de la technologie qui aurait même pour d'effet de contribuer au chồmage. Sur ce ton, les auteurs concluent - en citant Alvin Toffler qui réfère à Karl Marx - que le leadership pour effectuer la transition relève... du lecteur.

\section{Appréciation}

Il faut savoir gré a Don Tapscott et à Art Caston d'avoir articulé un ensemble de shifts, de transferts, de transitions, de transits, de changements et de tenter d'incorporer ceux-ci sous la forme de deux modèles synthétiquement illustrés (initialement aux figures $1-2$ et $8-1$ ). Ils guident relativement bien le lecteur dans un labyrinthe organisationnel - informationnel - technologique hautement complexe. Les deux auteurs de Paradigm Shift ont su réunir et discuter d'un nombre considérable de sujets reliés à ce qui est communément nommé la technologie de l'information. Le fait d'avoir rassemblé autant de considérations technologiques, informationnelles et organisationnelles en 
quelque trois cents pages est méritoire. Cependant, il y a danger! «Qui trop embrasse, mal étreint " dit le proverbe. Voilà qui fait de cet ouvrage un livre pour lecteurs avertis.

Si le lecteur-spécialiste risque d'être enchanté par le déroulement des présentations, le lecteur-manager à qui le livre est également destiné devrait, à juste titre, s'interroger sur l'ordre dans lequel la matière lui est étalée. On a l'impression qu'il faut changer, car la technologie permet de le faire. De fait, le lecteur-manager qui ne prend pas garde risque de recevoir des messages du genre : « Hâtez-vous à changer votre organisation, car la nouvelle technologie rend cela possible. » Ou encore : «Voici les solu-

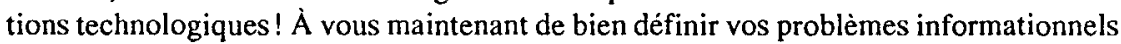
et organisationnels en conséquence, surtout si vous voulez demeurer compétitif et... survivre dans ce monde réel et cruel! ». Bref, il ne faut pas que le lecteur s'étonne si, à la suite de la lecture de quelques pages du livre, il estime que les auteurs ont "placé la charrue devant le bœuf ". Afin de véritablement comprendre et apprécier Paradigm Shift, il faut qu'il se rende jusqu'au bout et cela aussi rapidement que les capacités de son micro-ordinateur situé entre ses deux oreilles le permettent. La lecture de type paragraphe par paragraphe, répartie sur plusieurs semaines, risque d'offrir une vision hétéroclite du domaine traité et de mal interpréter le message. Faut-il croire que le sujet est trop intégré pour faire autrement?

Il convient de reprocher aux auteurs d'être tombé trop rapidement, c'est-à-dire, après quelques pages, dans le panneau des solutions technologiques - route facile et impressionnante! - et de ne pas s'être attardé davantage, dès les débuts de l'ouvrage, sur une comparaison plus exhaustive entre l'organisation de l'ère I et celle de l'ère II. Comme il aurait été souhaitable, mais aussi captivant, de lire à la première partie du livre tout ce qui est organisationnel et managériel : aucune informatique, aucun ordinateur, mais plutôt des exposés, des discussions relatives uniquement au paradigme de l'organisation (l'importance de son intégration, de son extension, de ses problèmes face au monde nouveau) et son évolution en termes de transferts (shifts) à caractère strictement organisationnel et managériel ; quitte à laisser les dirigeants et leurs managers le soin de juger par eux-mêmes si leur organisation est viable ou non, $s$ 'ils doivent la changer ou pas. Nous comprenons que la technologie impose et qu'elle peut agir comme catalyseur de changement. Même si tout est intégré, il ne faut pas donner - surtout dans les débuts! - l'impression qu'il faut changer pour changer. Cette argumentation a toujours été faible; il n'y a pas encore eu de shift à cet égard.

Autre reproche, plus doux celui-ci, touche l'orientation business du livre... typiquement américain, quoi! Les auteurs citent en exemple et réfèrent à plusieurs organisations: American Airlines, Citicorp, American Home Shield, National Car Rental, Hertz, Coke (Tokyo), American Express, TransAlta Utilities Corp., Noranda, Inc., The Travelers, Federal Express, Northern Telecom, Otis Elevator, Toys 'R' Us, Sears Roebuck, Levi Strauss, Shell, etc. Nous comprenons que pour devenir un best-seller cet ouvrage doit se vendre aux États-Unis et que là-bas, tout est business! Or, même s'il faut reconnaître que quelques cas sont tirés du secteur public (22 saisies des mêmes données dans un ministère anonyme [p. 61]), l'impression trop souvent avancée est que l'ouvrage ne vise que l'entreprise privée - business - le secteur privé. Néanmoins, le lecteur de l'administration publique, qu'il soit orienté vers l'élaboration de politiques ou vers les tâches managérielles risque d'y tirer parti. Bref, l'emploi plus répandu et 
sélectif du nom et de l'adjectif organization aurait su communiquer une association, une interprétation, une transposition plus faciles quant aux leçons à retenir par les ministères et les organismes des secteurs public, parapublic et péripublic. (Loin de nous l'idèe de suggérer aux auteurs de référer, de discuter même de cas ou d'exemples québécois... Ce livre doit se vendre à New York et ailleurs!)

Quoi de neuf dans ce livre ? Le lecteur diplômé depuis les cinq dernières années (sinon davantage) de facultés ou d'écoles d'administration publique ou privée et de départements d'informatiques y verront une belle synthèse des enseignements qui leur ont été offerts par les modules des SI. Contrairement aux prétentions exprimées lors des premières phrases des auteurs, ils - et DMR! - n'ont pas tout inventé. Mais il ne faut pas leur en vouloir. La compétition est féroce! Nous ne référons pas aux recherches et aux enseignements universitaires, mais plutôt aux autres firmes de consultants! $\dot{A}$ titre d'exemple, Edward Yourdon, le président de la célèbre firme new-yorkaise (qui porte son nom) vient tout juste de décrire le World-class software organization dans un livre qui est plus orienté vers la programmation et le développement de logiciels (Decline \& Fall of the American Programmer, Englewood Cliffs, New Jersey: Yourdon Press, 1992). Il y a aussi l'ouvrage de Patrick Smith, le directeur de la technologie chez SHL Systemhouse, qui discute de l'informatique "client/serveur " et où l'on peut lire dès la première phrase de l'avant-propos : «Un transfert (shift) fondamental est en voie de se réaliser quant à la façon dont les businesses se servent des systèmes d'information pour leur avantage compétitif " (Client/Server Computing, Carmel, Indiana: SAMS Publishing, 1992). Combien d'autres livres de ce genre - la compétition aidant! - sont maintenant disponibles ou paraîtront sous peu...

Rolland Hurtubise

École nationale d'administration publique

Training at Work: A Critical Analysis of Policy and Practice, by Jeff Hyman, London, Routledge, 1992, 191 p., ISBN 0-415-05343-9.

Worker training has taken on particular importance at the present time given a variety of forces: globalization and specialization in world markets, changing organizational structures, new ways of organizing work, higher skills required at entry level positions, new technologies, changes in the labour force, professionnel obsolescence. Training should be seen as a mechanism for investing in human capital to assist a country in improving its competitiveness.

Although the title of this volume may give the impression that the book is a training manual or a compendium of training techniques, this is not the case. Hyman's book is not a comprehensive and practical guide to the employee training process (i.e. training needs assessment, training design, training methods, maximizing trainees learning, evaluating training program). The volume by Wexley and Latham (1991) or the extensive updated review by Goldstein (1992) represent more comprehensive treatments of the training process. 